\title{
Integrated control of direct yaw moment control and active suspension system for $4 w d$ vehicles
}

\author{
Jianjun Hu*,**, Yi Han**, Jia Chen** \\ *State Key Laboratory of Mechanical Transmission, Chongqing University, Chongqing 400044, China, \\ E-mail: hujianjun@cqu.edu.cn \\ **School of Automotive Engineering, Chongqing University, Chongqing 400044, China
}

cross'ref http://dx.doi.org/10.5755/j01.mech.23.3.18481

\section{Introduction}

Recently, due to the increasing needs of active safety systems, various chassis control systems have been introduced in the automotive industry. Such as ABS (antilock braking system), ESP (electronic stability program), AFS (active front steering), VDC (vehicle dynamics control system), ASS, DYC and ARS (active roll control system) are being applied to achieve greater performance. Dongshin [1] developed the MANDO ESP, and study the performance of ESP system in the emergency situation such as the final oversteer and understeer. Frequently, these chassis control systems are always designed and developed independent of each other, even though their dynamics often interact. For future prospects, the effect of chassis control systems can be further increased by the integration of those chassis control systems.

Most of researchers focus on the integrated control system in order to improve the comprehensive performance of vehicle. Marino [2] has a further research in the integrated control of front and rear active differentials with AFS in order to improve dynamics and stability. In the thesis [3] an Integrated Chassis Control of ESC (Electronics Stability Control), 4WD and ARS (Active Roll Control System) is presented to improve high speed cornering performance. In order to handle critical driving situations by activating several subsystems (semi-active suspensions, active steering and electro-mechanical braking actuators) in a hierarchical, in [4] proposes a new multivariable LPV=H1 (Linear Parameter Varying) control strategy for GCC (global chassis control). An integrated chassis control algorithm of differential braking, front/rear traction torque, and active roll moment control is presented in [5]. An improved inverse Nyquist array based feedback ICC controller is designed for AFS and ESC integration to decouple the plant dynamics under different frequency bands [6]. In [7], linear quadratic control theory has been applied to the design the integrated control of DYC and AFS.

At present, the research of integrated control for vehicle chassis mainly concentrates in AFS and DYC, AFS and ESC. The study of stability integration control for 4WD vehicle equipped with active suspension system to improve vehicle comprehensive performance is few. In this study, based on understeer and oversteer condition, the integrated control of DYC and ASS for a full-time four-wheel drive vehicle is studied. And the proposed integrated control strategy is verified. This paper consists of five sections. The second section presents the Dynamics model of 4WD vehicle. In the third section, a sliding mode controller for DYC and a PID controller for ASS are given. Then an integrated controller is designed to coordinate DYC and ASS. The simulation results and conclusion are presented in the last two sections.

\section{Dynamics model of 4WD vehicle}

The research object is a medium 4WD vehicle model equipped with electronic control gasoline engine. The powertrain mainly includes clutch, transmission, limited slip differential, transmission shaft, inter-wheel torque distribution device (active differential) and wheel [8]. The structure is shown in Fig. 1.

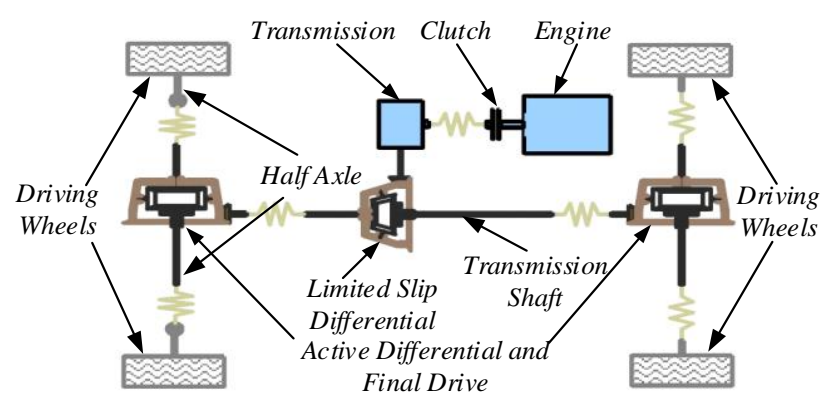

Fig. 1 Structure of $4 \mathrm{WD}$ vehicle

Torque distribution characteristic of the active differential

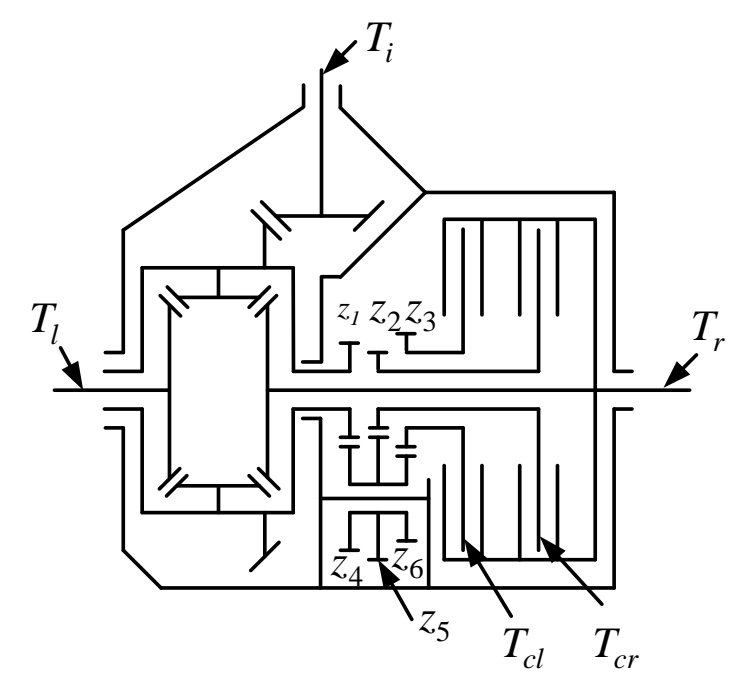

Fig. 2 The schematic diagram of the inter-wheel torque distribution device 
The structure of inter-wheel torque distribution device is shown in Fig. 2. Two friction clutches (left clutch and right clutch) and a set of triple gear driver are added to the traditional differential. Through engaging different clutches, the transfer of driving torque is realized. When the right clutch engages, the torque of half axle on right side is increased and torque is transmitted by speed shaft. When the left clutch engages, the torque of half axle on left side is increased and torque is transmitted by reduction shaft.

When the right clutch engages, the driving torque of left and right wheels can be derived as follow:

$$
\left\{\begin{array}{l}
T_{l}=\frac{T_{i}}{2}-\frac{z_{1} z_{5}}{2 z_{2} z_{4}} T_{c r}, \\
T_{r}=\frac{T_{i}}{2}+\left(1-\frac{z_{1} z_{5}}{2 z_{2} z_{4}}\right) T_{c r},
\end{array}\right.
$$

When the left clutch engages, the driving torque of left and right wheels can be derived as follow:

$$
\left\{\begin{array}{l}
T_{l}=\frac{T_{i}}{2}+\frac{z_{1} z_{6}}{2 z_{3} z_{4}} T_{c l}, \\
T_{r}=\frac{T_{i}}{2}-\left(1-\frac{z_{1} z_{6}}{2 z_{3} z_{4}}\right) T_{c l},
\end{array}\right.
$$

where $T_{i}$ is input torque; $T_{l}$ and $T_{r}$ are driving torque of left wheel and right wheel, respectively; $T_{c l}$ and $T_{c r}$ are friction torque of left clutch and right clutch, respectively; $z_{1}$ is the number of gear teeth for differential shell; $z_{4}$ is the number of gear teeth for clutch driving disc; $z_{2}$ is the number of gear teeth for right clutch transmission gear; $z_{3}$ is the number of gear teeth for left clutch transmission gear; $z_{4}, z_{5}$ and $z_{6}$ are the number of gear teeth for triple gear, respectively.

Transmission system model

The torque equilibrium equation of front and rear active differentials can be obtained as follow:

$$
\left\{\begin{array}{l}
J_{w} \dot{\omega}_{f 1}=\frac{1}{2} T_{f} i_{0} \eta_{0}-\left(F_{w x 1} r_{w}+f_{1} F_{z 1} r_{w}\right), \\
J_{w} \dot{\omega}_{f 2}=\frac{1}{2} T_{f} i_{0} \eta_{0}-\left(F_{w x 2} r_{w}+f_{2} F_{z 2} r_{w}\right), \\
J_{w} \dot{\omega}_{r 3}=\frac{1}{2} T_{r} i_{0} \eta_{0}-\left(F_{w x 3} r_{w}+f_{3} F_{z 3} r_{w}\right), \\
J_{w} \dot{\omega}_{r 4}=\frac{1}{2} T_{r} i_{0} \eta_{0}-\left(F_{w x 4} r_{w}+f_{4} F_{z 4} r_{w}\right), \\
\dot{\omega}_{f 1}+\dot{\omega}_{f 2}=2 \dot{\omega}_{o f}, \\
\dot{\omega}_{r 1}+\dot{\omega}_{r 2}=2 \dot{\omega}_{o r} .
\end{array}\right.
$$

The equation (3) can be rewrote as follow:

$$
\left\{\begin{array}{l}
J_{w} \dot{\omega}_{o f}=T_{f} i_{0} \eta_{0}-\left(F_{w x 1} r_{w}+f_{1} F_{z 1} r_{w}+F_{w x 2} r_{w}+f_{2} F_{z 2} r_{w}\right), \\
J_{w} \dot{\omega}_{o r}=T_{r} i_{0} \eta_{0}-\left(F_{w x 3} r_{w}+f_{3} F_{z 3} r_{w}+F_{w x 4} r_{w}+f_{4} F_{z 4} r_{w}\right),
\end{array}\right.
$$

limited slip differential $\left(T_{f}=\frac{T_{i}-T_{c}}{2}, T_{r}=\frac{T_{i}+T_{c}}{2}\right)$.

Above all, the input torque equation of limited slip differential is

$$
T_{i}=\frac{4 J_{w} T_{e} i_{g} i_{o d} \eta_{g}+i_{0} J_{e q} T_{x w}}{4 J_{w}+i_{0}^{2} \eta_{0} J_{e q}},
$$

where

$$
T_{x w}=F_{w x 1} r_{w}+F_{w x 2} r_{w}+F_{w x 3} r_{w}+F_{w x 4} r_{w}+
$$$$
+f_{1} F_{z 1} r_{w}+f_{2} F_{z 2} r_{w}+f_{3} F_{z 3} r_{w}+f_{4} F_{z 4} r_{w}
$$

$J_{e q}=J_{e} i_{g}^{2} i_{o d}^{2}+J_{g} i_{o d}^{2}+J_{o d} ; F_{w x 1}, F_{w x 2}, F_{w x 3}, F_{w x 4}$ are longitudinal force of front-right, front-left, rear-left and rearright driving wheels, respectively; $i_{g}, i_{\text {od }}, i_{0}$ are transmission ratio of transmission, active differential and final drive, respectively; $r_{w}$ is wheel radius; $F_{z 1}, F_{z 2}, F_{z 3}, F_{z 4}$ are dynamic load of front-right, front-left, rear-left and rearright wheels, respectively; $f_{1}, f_{2}, f_{3}, f_{4}$ are rolling resistance coefficient of front-right, front-left, rear-left and rear-right wheels, respectively; $\eta_{g}$ is transmission efficiency of engine to limited slip differential; $\eta_{0}$ is transmission efficiency of transmission shaft; $J_{e} \square J_{g} \square J_{o d} \square J_{w}$ are moment of inertia for engine, transmission, active differential, transmission shaft, final drive and wheel, respectively; $T_{c}$ is limited slip torque of limited slip differential.

Wheel model

In this study, the wheel mode is PAC2002. The "magic equation" is:

$$
\begin{aligned}
& Y(x)=D \sin [C \arctan \{B x-E(B x-\arctan B x)\}]+S_{v}, \\
& x=X+S_{h},
\end{aligned}
$$

where $Y$ is lateral force, longitudinal force or aligning force; $X$ is side-slip angle $\alpha$ or longitudinal slip rate $\lambda ; D$ is peak factor; $B$ is stiffness factor; $C$ is factor of curve shape; $E$ is factor of curve curvature; $S_{h}$ is horizontal drift of curve; $S_{v}$ is vertical drift of curve.

In addition to $C$, all the parameters are the functions of vertical load $F_{z}$, road adhesion coefficient $\mu$, wheel camber angle $\gamma$, side-slip angle $\alpha$ or longitudinal slip rate $\lambda$.

In the combined condition of driving/braking and steering, the relationships between wheel longitudinal force $F_{w x}$, lateral force $F_{w y}$ and side-slip angle $\alpha$, longitudinal slip rate $\lambda$, vertical load $F_{z}$ respectively are:

$$
F_{w x}=\frac{\sigma_{x}}{\sigma} F_{w x 0}, F_{w y}=\frac{\sigma_{y}}{\sigma} F_{w y 0},
$$

where $\sigma=\sqrt{\sigma_{x}^{2}+\sigma_{y}^{2}}, \sigma_{x}=-\frac{\lambda}{1+\lambda}, \sigma_{x}=-\frac{\tan \alpha}{1+\lambda}, F_{w x 0}$, $F_{w y 0}$ are longitudinal force and lateral force of driving, braking or steering condition, respectively. 


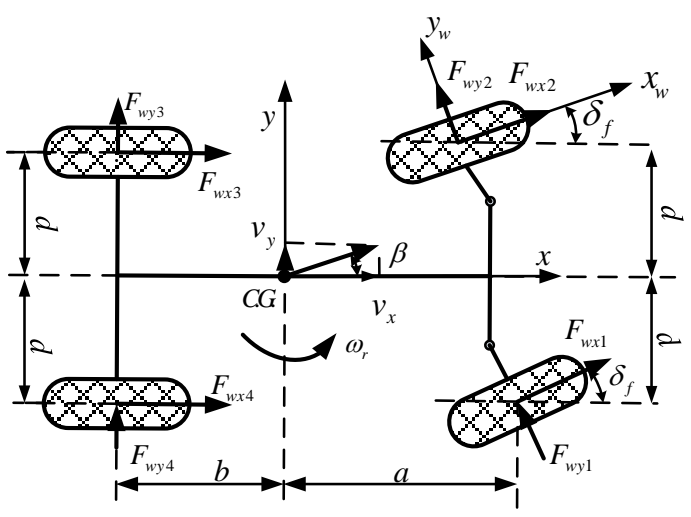

Fig. 3 The model of planar motion

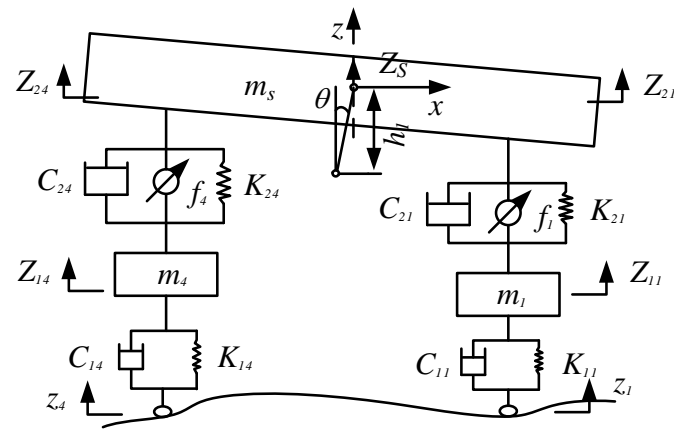

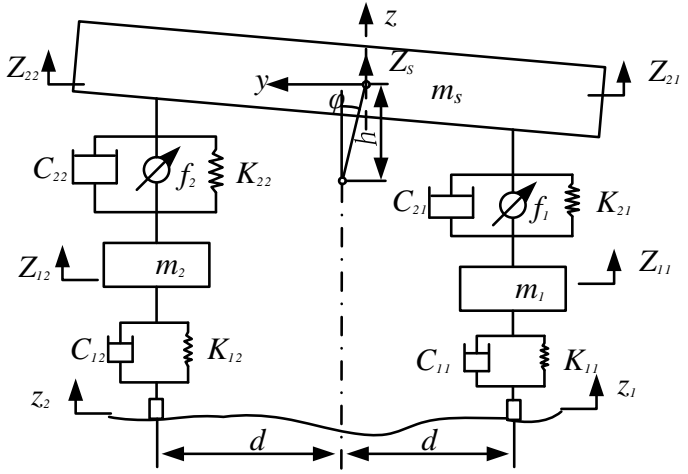

Fig. 5 The model of roll motion

Figs. 3-5 are the schematic diagrams of force analysis for vehicle. A non-linear and ten degree of freedom (DOF) vehicle model is set up, which includes longitudinal, lateral, vertical, yaw, roll and pitch directions and the vertical vibration DOF of 4 wheels [8].

The equations of forces and torques can be obtained as follow:

Longitudinal motion:

Fig. 4 The model of pitch motion

$$
m\left(\dot{v}_{x}-v_{y} \cdot \omega_{r}\right)+m_{s} h_{1} \ddot{\theta}=\sum F_{x}=\left(F_{w x 1}+F_{w x 2}\right) \cos \delta_{f}-\left(F_{w y 1}+F_{w y 2}\right) \sin \delta_{f}+\left(F_{w x 3}+F_{w x 4}\right)-m g f-\frac{C_{D} A_{x}}{21.15}\left(3.6 * v_{x}\right)^{2} .
$$

Lateral motion:

$$
m\left(\dot{v}_{y}+v_{x} \cdot \omega_{r}\right)-m_{s} h \ddot{\varphi}=\sum F_{y}=\left(F_{w y 1}+F_{w y 2}\right) \cos \delta_{f}+\left(F_{w x 1}+F_{w x 2}\right) \sin \delta_{f}+\left(F_{w y 3}+F_{w y 4}\right) .
$$

Vertical motion:

$$
m_{s} \ddot{Z}_{s}=\sum F_{z}=F_{22}+F_{23}+F_{21}+F_{24} .
$$

Yaw motion:

$$
\begin{aligned}
I_{z} \dot{\omega}_{r}=\sum M_{z}= & a\left[\left(F_{w y 1}+F_{w y 2}\right) \cos \delta_{f}+\left(F_{w x 1}+F_{w x 2}\right) \sin \delta_{f}\right]-b\left(F_{w y 3}+F_{w y 4}\right)+ \\
& \left.+d\left[\left(F_{w x 1}-F_{w x 2}\right) \cos \delta_{f}+\left(F_{w y 2}-F_{w y 1}\right) \sin \delta_{f}-F_{w x 3}+F_{w x 4}\right)\right] .
\end{aligned}
$$

Roll motion:

$$
I_{x} \ddot{\varphi}=\sum M_{x}=d\left[F_{22}+F_{23}-F_{21}-F_{24}\right]+m_{s} h\left(\dot{v}_{y}+v_{x} \cdot \omega_{r}\right)+m_{s} g h \varphi .
$$

Pitch motion:

$$
I_{y} \ddot{\theta}=\sum F_{z}=b\left(F_{23}+F_{24}\right)-a\left(F_{21}+F_{22}\right)-m_{s} h_{1}\left(\dot{v}_{x}-v_{y} \cdot \omega_{r}\right)+m_{s} g h_{1} \theta .
$$

Vertical motion of wheel:

$$
m_{1 i} \ddot{Z}_{1 i}=F_{1 i}-F_{2 i}-F_{a i} \quad(i=1,2,3,4)
$$

where $F_{a i}$ is additional vertical load of wheel, which is caused by inertia force of suspended mass; $F_{1 i}$ is vertical force of wheel; $F_{2 i}$ is acting force of suspension and

$$
\begin{aligned}
& F_{1 i}=K_{1 i}\left(z_{i}-Z_{1 i}\right)+C_{1 i}\left(\dot{z}_{i}-\dot{Z}_{1 i}\right), \quad(i=1,2,3,4) ; \\
& F_{2 i}=K_{2 i}\left(Z_{1 i}-Z_{2 i}\right)+C_{2 i}\left(\dot{Z}_{1 i}-\dot{Z}_{2 i}\right)+f_{i}, \quad(i=1,2,3,4) .
\end{aligned}
$$



small, so:

Pitch angle $\theta$ and roll angle $\varphi$ are considered to be

$$
\begin{aligned}
& Z_{21}=Z_{s}-a \theta-d \varphi, Z_{22}=Z_{s}-a \theta+d \varphi, \\
& Z_{23}=Z_{s}+b \theta+d \varphi, Z_{24}=Z_{s}+b \theta-d \varphi .
\end{aligned}
$$

The coupling between pitch motion and roll motion, front axle and rear axle is ignored. So the additional vertical loads of four wheels can be derived as follow:

$$
\left\{\begin{array}{c}
F_{a 1}=-\frac{m_{s} a_{x} h_{p}}{2 l}+\frac{m_{s} a_{y} h_{f}}{2 d} \cdot \frac{b}{l}, \\
F_{a 2}=-\frac{m_{s} a_{x} h_{p}}{2 l}-\frac{m_{s} a_{y} h_{f}}{2 d} \cdot \frac{b}{l}, \\
F_{a 3}=\frac{m_{s} a_{x} h_{p}}{2 l}-\frac{m_{s} a_{y} h_{r}}{2 d} \cdot \frac{a}{l}, \\
F_{a 4}=\frac{m_{s} a_{x} h_{p}}{2 l}+\frac{m_{s} a_{y} h_{r}}{2 d} \cdot \frac{a}{l} .
\end{array}\right.
$$

The longitudinal acceleration and lateral acceleration of vehicle are:

$$
a_{x}=\left(\dot{v}_{x}-v_{y} \omega_{r}\right)+h_{1} \ddot{\theta}, a_{y}=\left(\dot{v}_{y}+v_{x} \omega_{r}\right)-h \ddot{\varphi} .
$$

In wheel model, the vertical load should be considered. The vertical loads of four wheels can be obtained as follow:

$$
\begin{aligned}
& F_{z 1}=\frac{m g b}{2 l}+F_{11}, \quad F_{z 2}=\frac{m g b}{2 l}+F_{12}, \\
& F_{z 3}=\frac{m g a}{2 l}+F_{13}, \quad F_{z 4}=\frac{m g a}{2 l}+F_{14} .
\end{aligned}
$$

In equation (19), the first part is static load of each wheel and the second part is dynamic load of each wheel, which can be obtained by equation (15), where $m, m_{s}$, $m_{1 i}$ are gross mass, suspended mass and non-suspended mass, respectively; $v_{x}, v_{y}$ are vehicle longitudinal velocity and lateral velocity, respectively; $\omega_{r}, \dot{\omega}_{r}, \varphi, \ddot{\varphi}, \theta$, $\ddot{\theta}$ are vehicle yaw angular velocity, yaw angular acceleration, roll angle, roll angular acceleration, pitch angle, pitch angular acceleration, respectively; $h, h_{1}$ are height of spring mass center to roll center and pitch center, respectively; $F_{w x i}, F_{w y i}, F_{z i}$ are wheel longitudinal force, lateral force and dynamic load, respectively; $\delta_{f}$ is steering angle of front wheel; $g$ is gravity acceleration; $f$ is wheel rolling resistance coefficient; $C_{D}$ is air resistance coefficient; $A_{x}$ is windward area of vehicle; $Z_{s}$ is vertical displacement of suspended mass center; $I_{x}, I_{y}, I_{z}$ are moment of inertia for roll motion, pitch motion and yaw motion, respectively; $a, b$ are distance of vehicle mass center to front and rear axles, respectively; $d$ is half-axle-track; $Z_{1 i}$ is vertical displacement of non-suspended mass; $Z_{2 i}$ is vertical displacement of suspended mass which non-suspended mass corresponds to; $K_{2 i}, C_{2 i}, f_{i}$ are suspension stiffness, damping and acting force, respectively; $K_{1 i}, C_{1 i}$ are wheel stiffness and damping, respectively; $l$ is wheel base, $l=a+b ; h_{p}$ is vertical height of pitch center; $h_{f}, h_{r}$ are vertical height of the front and rear roll axles, respectively.

\section{Integrated controller design}

3.1. Nominal value of control variables

Based on the linear and two DOF vehicle model, the nominal value of vehicle yaw angular velocity $\omega_{r}$ and side-slip angle of mass center $\beta$ can be obtained.

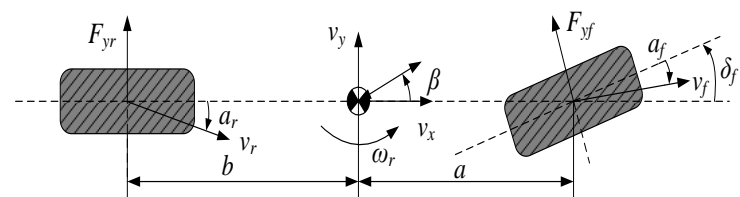

Fig. 6 The model of two DOF vehicles

The two DOF vehicle model is shown in Fig. 6 . And its equation is:

$$
\left\{\begin{array}{l}
m v_{x}\left(\dot{\beta}+\omega_{r}\right)=F_{y f}+F_{y r}, \\
I_{z} \dot{\omega}_{r}=a F_{y f}-b F_{y r} .
\end{array}\right.
$$

When side-slip angle of wheel is small, the relationship between wheel lateral force and the corresponding side-slip angle is considered to be linear:

$$
\left\{\begin{array}{l}
F_{y f}=k_{f} \alpha_{f}, F_{y r}=k_{r} \cdot \alpha_{r}, \\
\alpha_{f}=\beta+\frac{a \omega_{r}}{v_{x}}-\delta_{f}, \alpha_{r}=\beta-\frac{b \cdot \omega_{r}}{v_{x}} .
\end{array}\right.
$$

With Eq. (20) and Eq. (21), the ideal model of vehicle steering motion can be derived as follow:

$$
\left\{\begin{array}{l}
\dot{\beta}=\frac{\left(k_{f}+k_{r}\right)}{m v_{x}} \beta+\left(\frac{a k_{f}-b k_{r}}{m v_{x}^{2}}-1\right) \omega_{r}-\frac{k_{f}}{m v_{x}} \delta_{f}, \\
\dot{\omega}_{r}=\frac{\left(a k_{f}-b k_{r}\right)}{I_{z}} \beta+\frac{\left(a^{2} k_{f}+b^{2} k_{r}\right)}{I_{z} v_{x}} \omega_{r}-\frac{a k_{f}}{I_{z}} \delta_{f} .
\end{array}\right.
$$

The nominal value of vehicle yaw angular velocity $\omega_{r m}$ and side-slip angle of mass center $\beta_{m}$ can be calculated, according to the formula (22).

\subsection{DYC controller design}

The additional yaw moment $M_{z}$ is offered by DYC controller to make the nominal value of yaw angular velocity and side-slip angle of mass center tracked well in the steering condition. [10]

The state variable $x$, input variable $u$ and output variable $y$ are defined as $\left[\begin{array}{ll}\beta & \omega_{r}\end{array}\right]^{T}, M_{z}$ and $\left[\begin{array}{ll}\beta & \omega_{r}\end{array}\right]^{T}$, respectively. The DYC can be described as follow: 


$$
\left\{\begin{array}{l}
\dot{x}=A x+B u+Q \delta_{f}, \\
y=C x
\end{array}\right.
$$

where $\quad A=\left[\begin{array}{ll}a_{11} & a_{12} \\ a_{21} & a_{22}\end{array}\right]=\left[\begin{array}{cc}\frac{\left(k_{f}+k_{r}\right)}{m v_{x}} & \left(\frac{a k_{f}-b k_{r}}{m v_{x}^{2}}-1\right) \\ \frac{\left(a k_{f}-b k_{r}\right)}{I_{z}} & \frac{\left(a^{2} k_{f}+b^{2} k_{r}\right)}{I_{z} v_{x}}\end{array}\right] ;$ $B=\left[\begin{array}{c}0 \\ \frac{1}{I_{z}}\end{array}\right] ; \quad C=\left[\begin{array}{cc}1 & 0 \\ 0 & 1\end{array}\right] ; \quad Q=\left[\begin{array}{l}q_{1} \\ q_{2}\end{array}\right]=\left[\begin{array}{c}-\frac{k_{f}}{m v_{x}} \\ a k_{f} \\ -\frac{I_{z}}{2}\end{array}\right]$.

$$
\dot{s}=\dot{\omega}_{r m}-a_{21} \beta-a_{22} \omega_{r}-q_{2} \delta_{f}-\frac{M_{z}}{I_{z}}-\varepsilon\left(\dot{\beta}_{m}-a_{11} \beta-a_{12} \omega_{r}-q_{1} \delta_{f}\right) .
$$

Constant speed reaching law is adopted in sliding mode control.

$$
\dot{s}=-k \operatorname{sgn}(s), \quad k>0,
$$

where $k$ is a constant and it stands for the approach velocity of system moving point to sliding surface. The smaller the $k$, the slower the approach velocity. The greater the $k$, the faster the approach velocity, but the vibration is large.

In order to avoid the vibration caused by sign func-
The target yaw moment control system is designed with sliding mode variable structure control algorithm. The sliding surface $(\mathrm{s}=0)$ is defined as the weighting of yaw angular velocity deviation $\left(e_{1}=\omega_{r m}-\omega_{r}\right)$ and mass center side-slip angle deviation $\left(e_{2}=\beta_{m}-\beta\right)$.

$$
s=\omega_{r m}-\omega_{r}-\varepsilon\left(\beta_{m}-\beta\right),
$$

where $\varepsilon$ is adjustable weighting coefficient and it can be adjusted according to the road adhesion coefficient, which reflects the proportion of side-slip angle deviation of mass center during calculating additional yaw moment [7].

With Eq. (23), Eq. (24) can be rewrote as follow:

$$
M_{z}=I_{z}\left(\dot{\omega}_{r m}-\varepsilon \dot{\beta}_{m}+\left(\varepsilon a_{12}-a_{22}\right) \omega_{r}+\left(\varepsilon a_{11}-a_{21}\right) \beta+\left(\varepsilon q_{1}-q_{2}\right) \delta_{f}+k s a t(s)\right) .
$$

The target yaw moment $M_{z}$ needs to be converted as the variable quantity of longitudinal force for driving wheels. In this study, variable quantity of longitudinal force for driving shaft is produced by controlling the friction torque of active differential.

When vehicle is not braking, the rotating dynamics equation of wheel is:

$$
T_{d}=F_{x w} r_{w},
$$

where $F_{x w}$ is driving force of wheel.

For instance, the relationship between longitudinal force difference of rear driving wheels and produced yaw moment is:

$$
M_{z}=\Delta F_{x w} d=\left(F_{x w r r}-F_{x w r l}\right) d \text {. }
$$

So the driving torque difference of driving wheels caused by active differential is:

$$
\Delta T_{d}=\left(F_{x w r r}-F_{x w r l}\right) r_{w}=\frac{M_{z} r_{w}}{d}
$$

Because of the clutch structure limitation, friction torque has to be limited. So $\left|\Delta T_{d}\right|$ must be less than $\Delta T_{d \max }$. tion, the sign function in equation (26) is replaced as saturation function [11]:

$$
\operatorname{sat}(s)=\left\{\begin{array}{ll}
\frac{s}{\tau} & |s| \leq \tau \\
\operatorname{sgn}(s) & |s|>\tau
\end{array},\right.
$$

where $\tau$ is boundary layer thickness. It is a positive and small constant, so $\tau$ is considered to be 0.01 in this study. With (25), (26) and (27), the target yaw moment:

When the right clutch engages:

$$
T_{c r}=\Delta T_{d}=\frac{M_{z} r_{w}}{d}
$$

When the left clutch engages:

$$
T_{c l}=\Delta T_{d}=\frac{M_{z} r_{w}}{d}
$$

The driving force difference of wheels on both sides is produced by transferring the driving torques of both sides of wheels with active differential.

Vehicle stability control is always working during vehicle sharp turn or large steering angle condition. In order to have enough steering ability, driving torque is mainly distributed to rear axle in $4 \mathrm{WD}$ vehicle. In addition, as steering angle of front wheels, the yaw moment of front axle is less than the one of rear axle with the same friction torque of active differential. And driver steering operation experience will be influenced by the fiction torque of front axle. Above all, in order to make DYC controller have a larger working range and decrease the influence on driver, the driving force distribution of rear axle should be considered primarily.

Control strategy of driving force distribution is shown in Table 1. 
Control strategy of driving force distribution

\begin{tabular}{|c|c|c|c|c|}
\hline Front wheel steering angle & $\Delta \omega_{r}=\omega_{r}-\omega_{r m}$ & $\begin{array}{c}\text { Steering } \\
\text { characteristic }\end{array}$ & $\begin{array}{c}\text { Torque transfer } \\
\text { direction }\end{array}$ & Clutch \\
\hline$\delta_{f}>0$ & $\Delta \omega_{r}<-\omega_{r t h}$ & Understeer & Right wheel & Right clutch engages \\
\hline$\delta_{f}>0$ & $\Delta \omega_{r}>\omega_{r t h}$ & Oversteer & Left wheel & Left clutch engages \\
\hline$\delta_{f}<0$ & $\Delta \omega_{r}>\omega_{r t h}$ & Understeer & Left wheel & Left clutch engages \\
\hline$\delta_{f}<0$ & $\Delta \omega_{r}<-\omega_{r t h}$ & Oversteer & Right wheel & Right clutch engages \\
\hline Arbitrary $\delta_{f}$ & $\left|\Delta \omega_{r}\right|<\omega_{r t h}$ & Neutral steer & - & - \\
\hline
\end{tabular}

\subsection{Active suspension system controller design}

Vehicle vibration includes vibration of unspring mass with uneven road and vibration of spring mass inertia. As the two different vibrations, double-loop control structure is adopted as Fig. 7 [12]. For the vibration with uneven road, four PD controllers are designed to decrease the vibration of the junction of suspension and vehicle body. The relative displacement change rate of each wheel corresponding junction of chassis and vehicle body $\left(\Delta \dot{Z}_{C i}=\dot{Z}_{2 i}-\dot{Z}_{1 i}\right.$ $(i=1,2,3,4))$ is input and the target control force of suspension system $\left(f_{1 i}(i=1,2,3,4)\right)$ is output. For the vibration of spring mass inertia, three PD controllers are designed to decrease vehicle vertical, roll and pitch motion. The vertical displacement of vehicle mass center $Z_{s}$, roll angle $\theta$ and pitch angle $\varphi$ are inputs and equivalent control force $F_{z}$, torque $M_{\theta}$ and $M_{\varphi}$ are outputs.
The relationships between suspension system control force $f_{2 i}$ and $F_{z}, M_{\theta}, M_{\varphi}$ respectively are:

$$
\begin{aligned}
& F_{z}=f_{21}+f_{22}+f_{23}+f_{24}, \\
& M_{\theta}=-a\left(f_{21}+f_{22}\right)+b\left(f_{23}+f_{24}\right), \\
& M_{\varphi}=d\left(-f_{21}+f_{22}+f_{23}-f_{24}\right) .
\end{aligned}
$$

The matrix form is:

$$
\left[\begin{array}{c}
F_{z} \\
M_{\theta} \\
M_{\varphi}
\end{array}\right]=A \times\left[\begin{array}{l}
f_{21} \\
f_{22} \\
f_{23} \\
f_{24}
\end{array}\right]=\left[\begin{array}{cccc}
1 & 1 & 1 & 1 \\
-a & -a & b & b \\
-d & d & d & -d
\end{array}\right]\left[\begin{array}{l}
f_{21} \\
f_{22} \\
f_{23} \\
f_{24}
\end{array}\right] .
$$

Matrix $A$ can't be inversed directly as it is not a square matrix. The target control force of four suspension systems $\left(f_{2 i}(i=1,2,3,4)\right)$ can be obtained with the generalized inverse matrix of matrix $A[12]$.

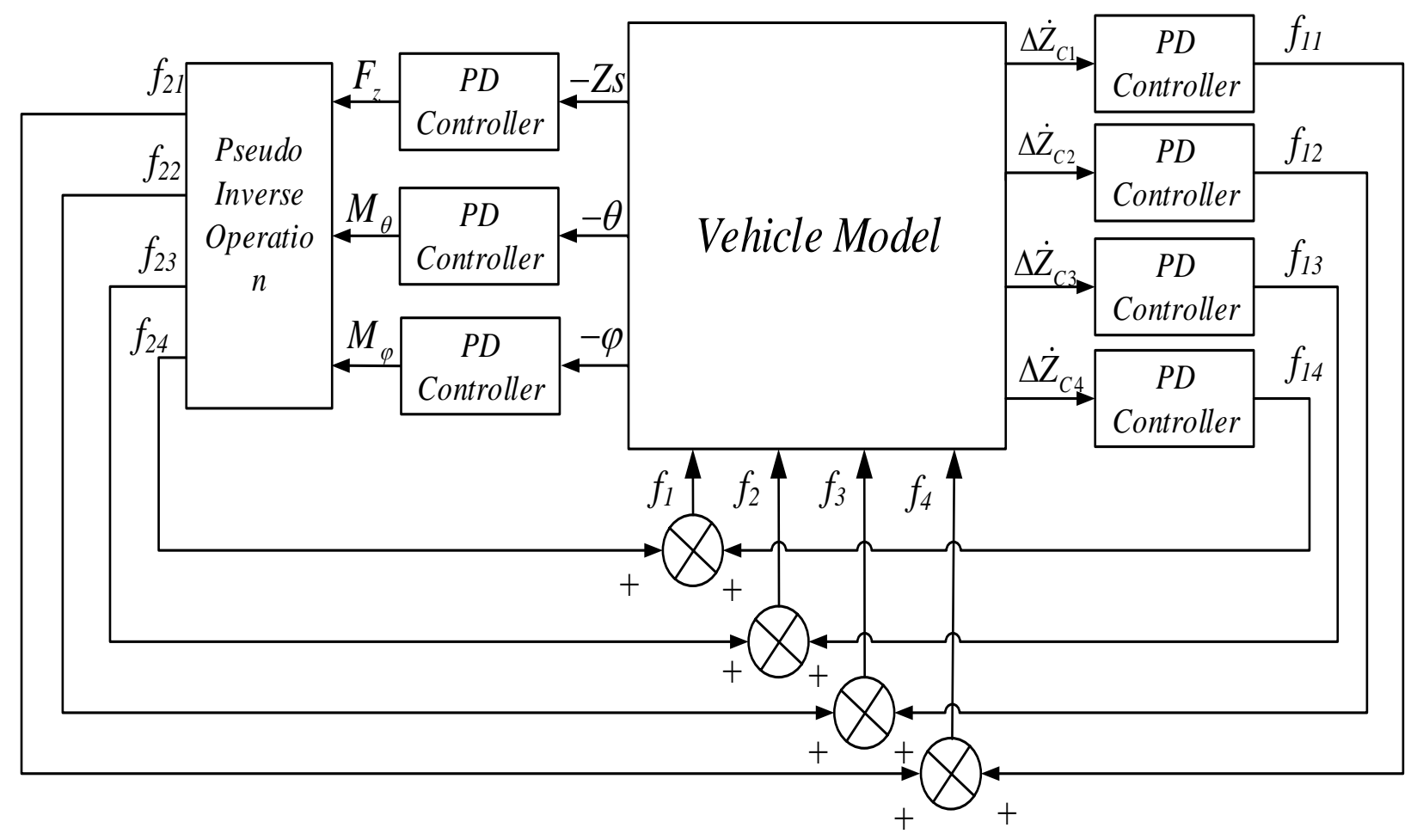

Fig. 7 Structure of active suspension system controller 


$$
\left[\begin{array}{l}
f_{21} \\
f_{22} \\
f_{23} \\
f_{24}
\end{array}\right]=\left[\begin{array}{ccc}
\frac{b}{2(a+b)} & \frac{-1}{2(a+b)} & \frac{-1}{4 d} \\
\frac{b}{2(a+b)} & \frac{-1}{2(a+b)} & \frac{1}{4 d} \\
\frac{a}{2(a+b)} & \frac{1}{2(a+b)} & \frac{1}{4 d} \\
\frac{a}{2(a+b)} & \frac{1}{2(a+b)} & \frac{-1}{4 d}
\end{array}\right]\left[\begin{array}{c}
F_{z} \\
M_{\theta} \\
M_{\varphi}
\end{array}\right] .
$$

The sum of control forces produced by double-loop controller is the suspension system control force:

$$
\left[\begin{array}{l}
f_{1} \\
f_{2} \\
f_{3} \\
f_{4}
\end{array}\right]=\left[\begin{array}{l}
f_{11} \\
f_{12} \\
f_{13} \\
f_{14}
\end{array}\right]+\left[\begin{array}{l}
f_{21} \\
f_{22} \\
f_{23} \\
f_{24}
\end{array}\right] .
$$

Displacement of vehicle mass center $Z_{s}$, roll angle $\varphi$ and pitch angle $\theta$ can be approximated as follow:

$$
\begin{aligned}
& Z_{S}=\frac{1}{4}\left(\Delta Z_{C 1}+\Delta Z_{C 2}+\Delta Z_{C 3}+\Delta Z_{C 4}\right), \\
& \theta=\frac{1}{a+b}\left(\frac{\Delta Z_{C 3}+\Delta Z_{C 4}}{2}-\frac{\Delta Z_{C 1}+\Delta Z_{C 2}}{2}\right), \\
& \varphi=\frac{1}{2 d}\left(\frac{\Delta Z_{C 2}+\Delta Z_{C 3}}{2}-\frac{\Delta Z_{C 1}+\Delta Z_{C 4}}{2}\right) .
\end{aligned}
$$

With the generalized inverse matrix in Eq. (36), Eq. (39) can be derived as follow:

$$
\left[\begin{array}{l}
f_{21} \\
f_{22} \\
f_{23} \\
f_{24}
\end{array}\right]=\left[\begin{array}{ccc}
\frac{b}{2(a+b)} & \frac{-1}{2(a+b)} & \frac{-k_{\varphi}}{2 d} \\
\frac{b}{2(a+b)} & \frac{-1}{2(a+b)} & \frac{k_{\varphi}}{2 d} \\
\frac{a}{2(a+b)} & \frac{1}{2(a+b)} & \frac{1-k_{\varphi}}{2 d} \\
\frac{a}{2(a+b)} & \frac{1}{2(a+b)} & \frac{k_{\varphi}-1}{2 d}
\end{array}\right]\left[\begin{array}{c}
F_{z} \\
M_{\theta} \\
M_{\varphi}
\end{array}\right],
$$

where $k_{\varphi}$ is distribution ratio coefficient of roll torque for front suspension and rear suspension $k_{\varphi} \in[0,1]$.

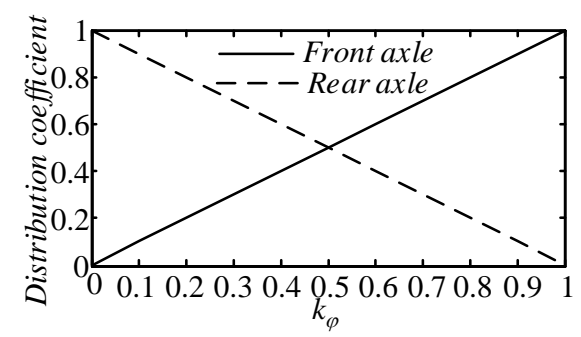

Fig. 8 Relationship between $k_{\varphi}$ and distribution coefficient of roll torque for front and rear axle

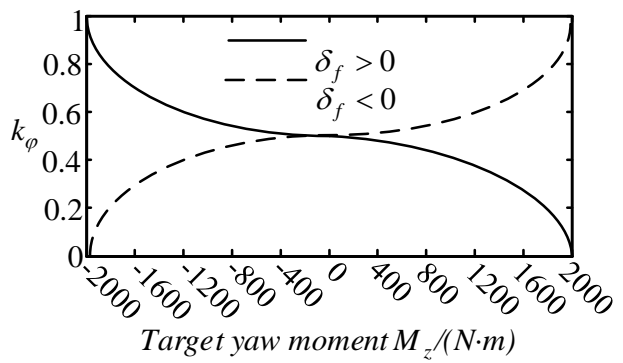

Fig. 9 Relationship between $k_{\varphi}$ and $M_{z}$

The relationship between $k_{\varphi}$ and distribution coefficient of roll torque for front axle and rear axle is shown in Fig. 8. When $k_{\varphi}$ is large, the roll torque of front axle is greater than the one of rear axle and the understeer tendency is increased. On the contrary, the less $k_{\varphi}$, the less cornering stiffness of rear axle and the oversteer tendency is increased.

The target of coordinating controller is to improve vehicle handling stability and guarantee vehicle posture by changing $k_{\varphi}$ value based on the judgment of vehicle driving cycle.

In section 3.2, the understeer or oversteer degree is reflected by the value of target yaw moment $M_{z}$ calculated by DYC controller, so $k_{\varphi}$ can be determined by $M_{z}$. In this study, define $x$ as $M_{z} / 4000+0.5$ and the equation of $k_{\varphi}$ is:

When $\delta_{f}$ is larger than zero,

$$
k_{\varphi}=\left\{\begin{array}{ll}
\frac{x-1+\sqrt{x-x^{2}}}{2 x-1} & x \neq 0.5 \\
0.5 & x=0.5
\end{array} .\right.
$$

When $\delta_{f}$ is less than zero,

$$
k_{\varphi}=\left\{\begin{array}{ll}
\frac{x-\sqrt{x-x^{2}}}{2 x-1} & x \neq 0.5 \\
0.5 & x=0.5
\end{array} .\right.
$$

The relationship between $k_{\varphi}$ and $M_{z}$ is shown in Fig. 9. When $M_{z}$ is small, the control system stability is principal and $k_{\varphi}$ changes slowly tracking $M_{z}$ to avoid fluctuation. When $M_{z}$ is large, the vehicle handling stability is principal and $k_{\varphi}$ changes quickly tracking $M_{z}$, which reflects that the understeer or oversteer degree is great.

\subsection{Integrated controller design}

The integrated control method of DYC and ASS is that the structure parameter of active suspension system controller can be adjusted by designing coordinating controller with DYC and ASS controller and the vehicle lateral stability can be improved by suspension system. 


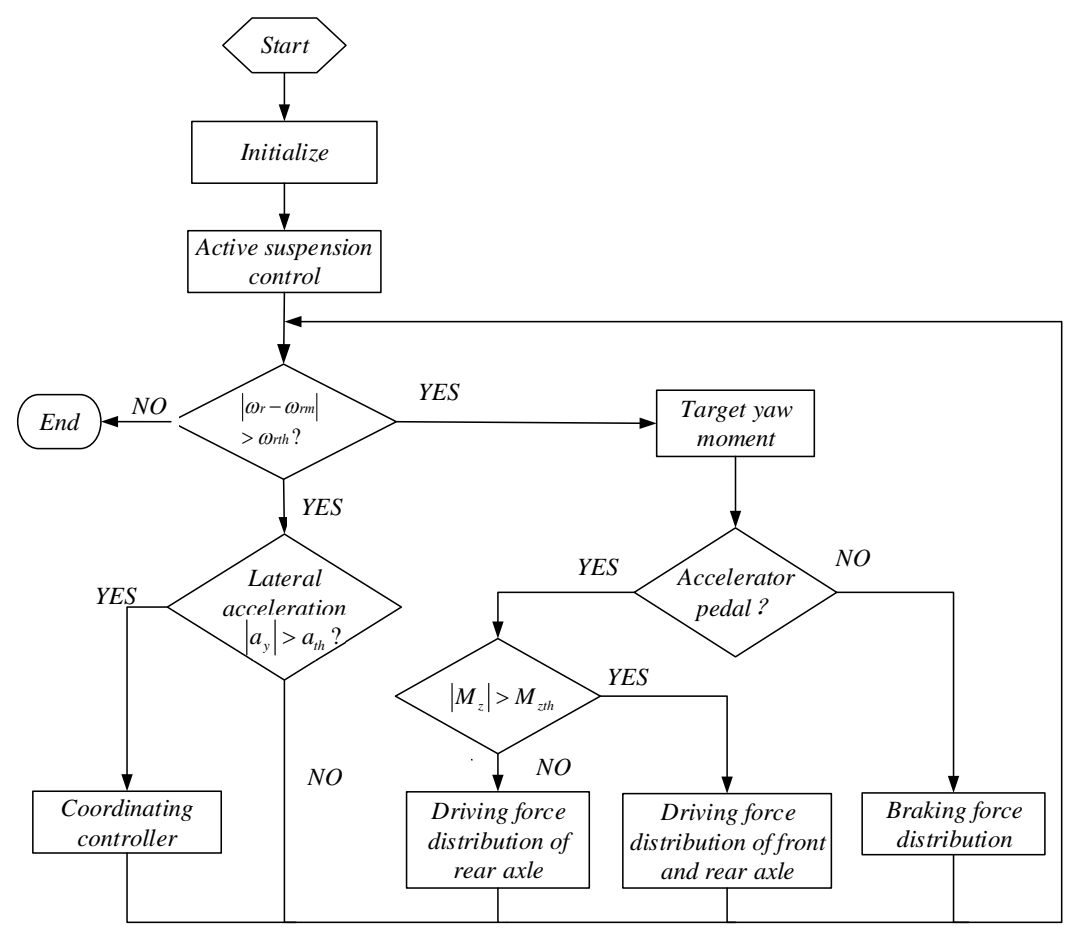

Fig. 10 Integrated control flowchart of DYC and ASS

Vehicle driving cycle should be judged first in integrated control. In linear driving cycle, only active suspension system should be controlled. In steering condition, it needs to be judged that active suspension system should be adjusted by coordinating controller according to the vehicle steering characteristic and lateral acceleration. Control flowchart is shown in Fig. 10. $\omega_{r t h}$ is threshold value which can prevent control system from starting when the difference between actual yaw angular velocity and expected one is small. When the absolute value of target yaw moment is less than threshold value $M_{z t h}$, only the driving force of rear axle is distributed. When the absolute value of target yaw moment is larger than threshold value $M_{z t h}$, the driving forces of front and rear axle are distributed. Integrated control strategy of DYC and ASS is shown in Table 2.

Table 2

Integrated control strategy of DYC and ASS

\begin{tabular}{|c|c|c|c|c|}
\hline \multirow{2}{*}{$\begin{array}{l}\text { Front wheel } \\
\text { steering angle }\end{array}$} & \multirow{2}{*}{$\Delta \omega_{r}=\omega_{r}-\omega_{r} n_{n}$} & \multirow{2}{*}{$\begin{array}{l}\text { Steering char- } \\
\text { acteristic }\end{array}$} & \multicolumn{2}{|c|}{ Integrated control strategy } \\
\hline & & & Direct yaw moment & Active suspension system(Roll torque) \\
\hline$\delta_{f}>0$ & $\Delta \omega_{r}<-\omega_{r t h}$ & Understeer & $\begin{array}{c}M_{z}>0 \\
\text { Right clutch engages }\end{array}$ & Distributed to rear axle \\
\hline$\delta_{f}>0$ & $\Delta \omega_{r}>\omega_{r t h}$ & Oversteer & $\begin{array}{c}M_{z}<0 \\
\text { Left clutch engages }\end{array}$ & Distributed to front axle \\
\hline$\delta_{f}<0$ & $\Delta \omega_{r}>\omega_{r t h}$ & Understeer & $\begin{array}{l}\qquad M_{z}<0 \\
\text { Left clutch engages }\end{array}$ & Distributed to rear axle \\
\hline$\delta_{f}<0$ & $\Delta \omega_{r}<-\omega_{r t h}$ & Oversteer & $\begin{array}{l}M_{z}>0 \\
\text { Right clutch engages }\end{array}$ & Distributed to front axle \\
\hline Arbitrary $\delta_{f}$ & $\left|\Delta \omega_{r}\right|<\omega_{r t h}$ & Neutral steer & - & - \\
\hline
\end{tabular}

Note: The positive direction of $M_{z}$ is clockwise direction based on vehicle driving direction.

General structure of integrated control with the control strategy of DYC and ASS is shown in Figure 11. For DYC controller, the additional yaw moment $M_{z}$ is calculated according to actual value and target value of control variables and it is transformed as the friction torque of active differential for front and rear axle $\Delta T_{d}$. For coordinating controller, the distribution ratio coefficient of roll torque for front suspension and rear suspension $k_{\varphi}$ is obtained according to vehicle lateral acceleration, steering angle direction of front wheels and $M_{z}$ to improve vehicle handling stability.

\section{Simulation results of integrated control}

To verify the feasibility of proposed integrated control strategy for DYC and ASS, the simulation model of integrated control for $4 \mathrm{WD}$ vehicle is established in the software of MATLAB/Simulink. The simulation of step steering condition and single lane change condition of vehicle is car- 
ried out. The simulation results of integrated control and independent control of DYC/ASS are analyzed.

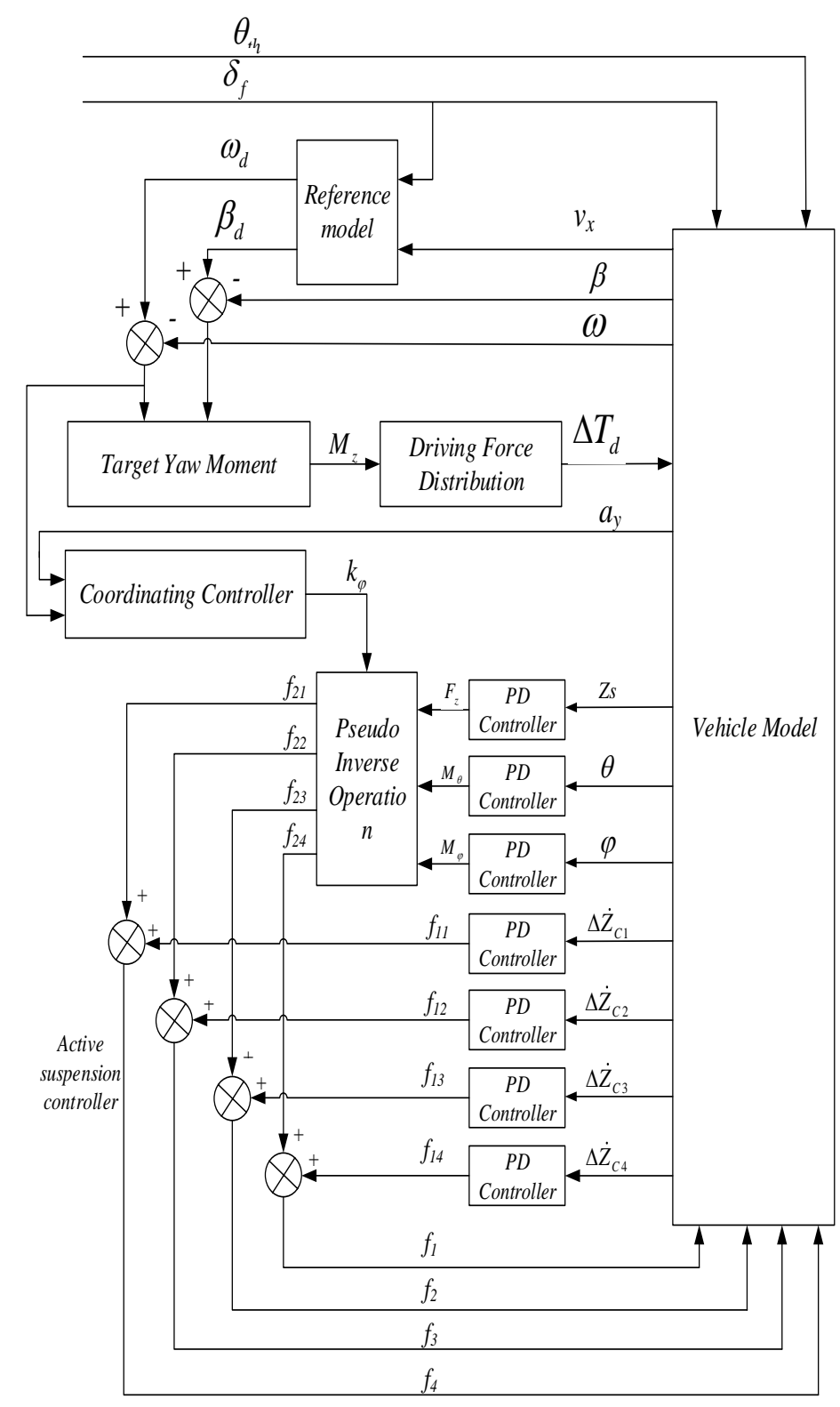

Fig. 11 General structure of integrated control

\subsection{The step steering condition}

The simulation conditions are as follow: set the road adhesion coefficient to 0.9 ; set the road irregularities input to 0 ; set the vehicle velocity to $90 \mathrm{~km} / \mathrm{h}$; the steering angle of front wheels is increased up to 4 degrees after $1 \mathrm{~s}$, and keeps unchanged till the end of simulation.

Fig. 12 refers to the results of simulation. With integrated control, the stable value of vehicle yaw angular velocity is a little larger than the one with independent control of DYC/ASS, and the vehicle traveling track is closer to the ideal one. But the same with independent control of DYC, the slid-slip angle of vehicle mass center is large with the increase of yaw angular velocity during integrated control. Compared with independent control, the fiction torque of active differential, velocity difference and driving force difference between left and right wheels are all decreased obviously with integrated control, and the friction torque of active differential is decreased 15.6 percent, which makes the potential of yaw moment produced by active differential developed and the friction consumption decreased.

Fig. 13 shows the ASS acting forces under step steering condition. With independent control of DYC/ASS, the force difference between left and right suspensions of front axle is $912 \mathrm{~N}$ and the one of rear axle is $930 \mathrm{~N}$ when vehicle is steady, as the roll torques of front and rear axles are distributed according to constant proportion law. The sum of force differences is $1842 \mathrm{~N}$. With integrated control, the force difference between left and right suspensions of front axle is $398 \mathrm{~N}$ and the one of rear axle is $1484 \mathrm{~N}$ when vehicle is steady, as the roll torques of front and rear axles are distributed according to coordinating control strategy. The sum of force differences is $1882 \mathrm{~N}$. Thus the designed integrated control can produce almost same roll torque, which makes the main control target of active suspension system not be influenced and the understeer of vehicle improved. 

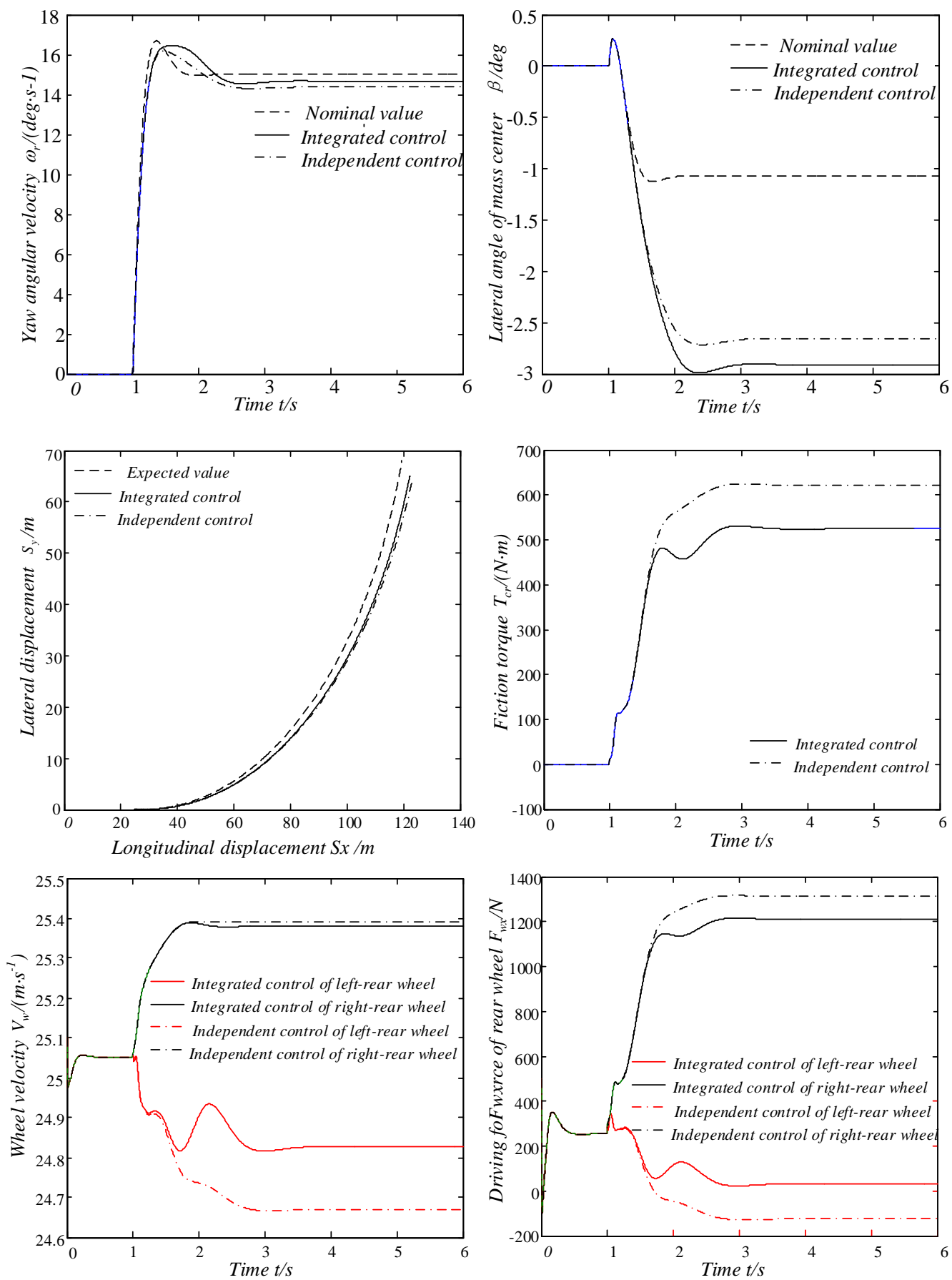

Fig. 12 Simulation results under step steering condition
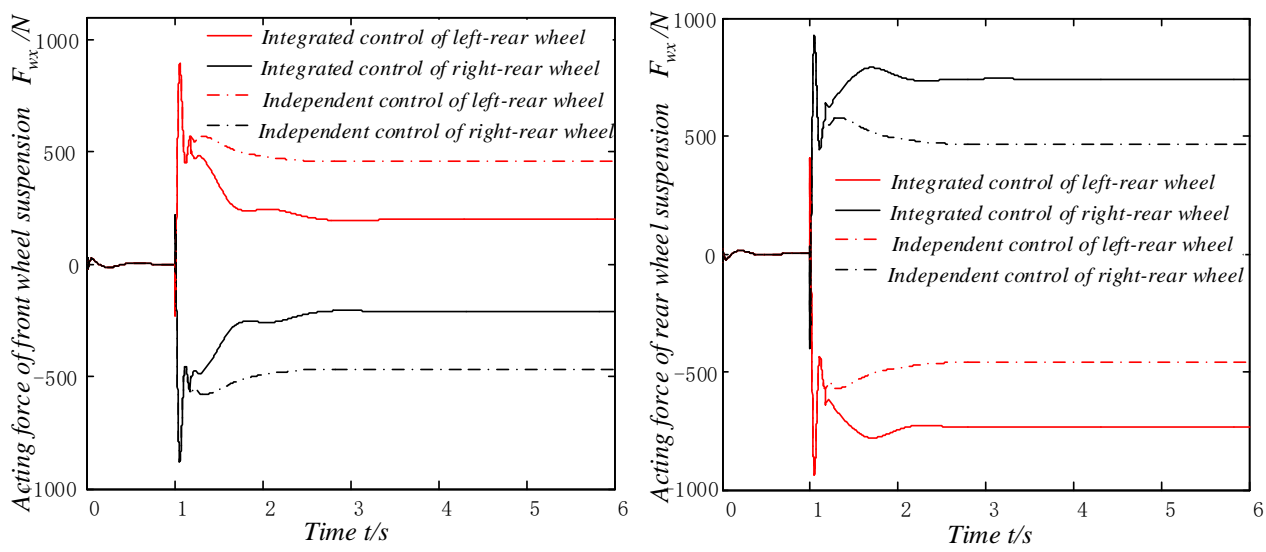

Fig. 13 ASS acting forces under step steering condition 


\subsection{The single lane change condition}

The simulation conditions are as follow: set the road adhesion coefficient to 0.9 ; set the vehicle velocity to $120 \mathrm{~km} / \mathrm{h}$; the steering angle of front wheels is calculated by means of driver model path tracking. The single lane change path is shown in Fig. 14.

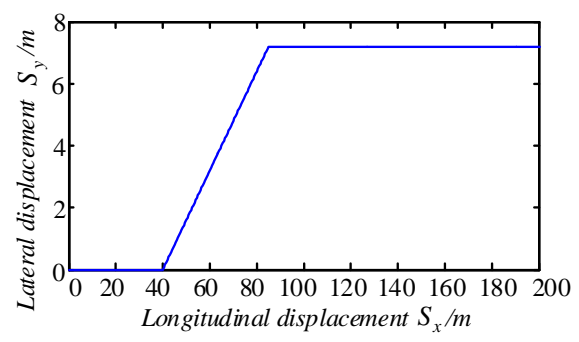

Fig. 14 Single lane change path
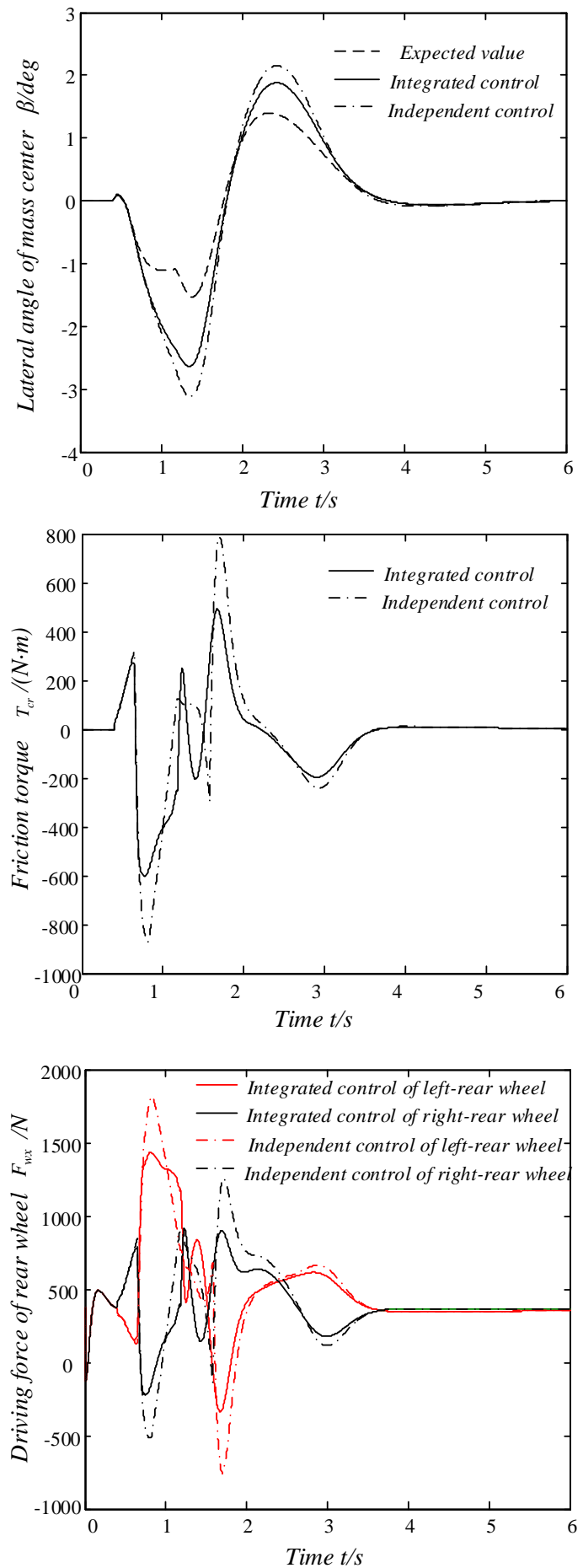

Fig. 15 Simulation results under single lane change condition

Fig. 15 refers to the results of simulation. The vehicle traveling track shows that vehicle has oversteer condition with large steering angle and high vehicle velocity. In integrated control, according to vehicle driving cycle, the cornering stiffness of front and rear axles is changed by ASS 
with roll torque distribution of front and rear axles to improve vehicle steering stability. Compared with independent control, although the vehicle traveling track of integrated control is near the one of independent control, the deviations between yaw angular velocity and nominal value, side-slip angle of mass center and nominal value are all decreased with integrated control. In addition, the friction torque of active differential and the driving force deviation between both sides of wheels are also decreased during integrated control, which makes the vehicle handling stability improved and DYC working range expanded.Fig. 16 shows the ASS acting forces under single lane change condition. The wheel load variable quantity of front axle is increased and the one of rear axle is decreased by coordinating controller with roll torque distribution at the beginning of steering. And the vehicle oversteer is partly improved based on that the vehicle posture isn't influenced.

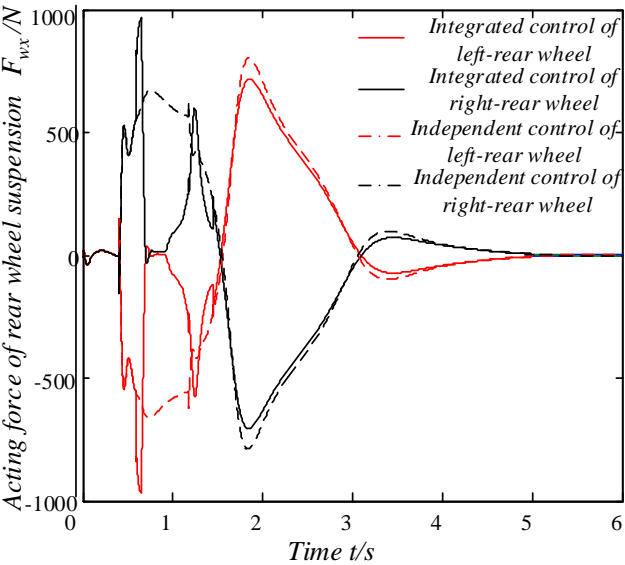

Fig. 16 ASS acting forces under single lane change condition

\section{Conclusions}

In this paper, A MF-Tire model that can reflect wheel non-linear and lateral-vertical coupling characteristics was adopted. A non-linear and ten degree of freedom vehicle dynamics model which can reflect the wheel characteristic was set up and it contained steering system and active suspension system. Based on the two degree of freedom vehicle model, DYC controller with driving torque distribution was designed by sliding mode variable structure control algorithm. As the vibrations caused by uneven road and inertia of spring mass, ASS controller with double-loop control structure was designed.

To improve vehicle riding comfort and handling stability during steering, the integrated control strategy of DYC and ASS was proposed according to the characteristic that wheel loads can be changed by force distribution of active suspension system. The simulation of step steering condition and single lane change condition of vehicle was carried out. The results indicate that the understeer or oversteer were prevented well with active driving torque distribution of rear wheels. The vehicle riding comfort and handling stability were both improved by the proposed integrated control strategy.

\section{References}

1. Kim, D.; Kim, K.; Lee, W.; Hwang, I. 2003. Development of Mando ESP (Electronic Stability Program). SAE 2003 World Congress \& Exhibition.

2. Marino, R.; Scalzi, S. 2009. Integrated Control of Active Steering and Electronic Differentials in Four Wheel Drive Vehicles. SAE Int. J. Passeng. Cars- Electron. Electr. Syst. 2(1): 141-149.

3. Heo, H.; Joa, E.; Yi, K.; and Kim, K. 2015. Integrated Chassis Control for Enhancement of High Speed Cornering Performance. SAE Int. J. Commer. Veh. 8(1):
102-109.

http://dx.doi.org/10.4271/2015-01-1568.

4. Fergani, S.; Sename, O.; Dugard, L. 2015. An LPV/Hœ integrated Vehicle Dynamic Controller. Vehicular Technology, vol.PP, no.99.

5. Her, H.; Koh, Y.; Joa, E.; Yi, K. 2015. An integrated control of differential braking, front/rear traction, and active roll moment for limit handling performance. IEEE Transactions on Vehicular Technology, 1-1.

6. Zhu, B.; Chen, Y.; Zhao, J. 2014. Integrated chassis control of active front steering and yaw stability control based on improved inverse nyquist array method. Thescientificworldjournal, 2014(3), 919847.

7. Nagai, M.; Shino, M.; Gao, F. 2002. Study on integrated control of active front steer angle and direct yaw moment. Jsae Review, 23(3), 309-315. http://dx.doi.org/10.1016/S0389-4304(02)00189-3.

8. Cairano, S. D.; Tseng, H. E.; Bernardini, D.; Bemporad, A. 2013. Vehicle yaw stability control by coordinated active front steering and differential braking in the tire sideslip angles domain. IEEE Transactions on Control Systems Technology, 21(4), 1236-1248. http://dx.doi.org/10.1109/TCST.2012.2198886.

9. Rajamani, R. 2011. Vehicle Dynamics and Control. Springer, New York, USA.

10. SHIBAHATA Chief Engineer, Y.; SHIMADA, K.; TOMARI, T. 1993. Improvement of vehicle maneuverability by direct yaw moment control. Vehicle System Dynamics, 22(5), 465-481.

11. Liu, X. Y. 2010. Research on Vehicle Stability Based on Direct Yaw Moment Control. Hefei University of Technology, Hefei, China.

12. Xiao, H.S.; Chen, W.W.; Zhou, H.H. 2009. Integrated vehicle dynamics control through coordinating electronic stability program and active suspension system. Mechatronics and Automation, Changchun, pp. 11501155 . 
Jianjun Hu, Yi Han, Jia Chen

INTEGRATED CONTROL OF DIRECT YAW MOMENT CONTROL AND ACTIVE SUSPENSION SYSTEM FOR 4WD VEHICLES

S u m m a r y

This paper presents an integrated control of DYC (direct yaw moment control system) and ASS (active suspension system) for 4WD (four-wheel drive) vehicles. In order to improve the comprehensive performance of $4 \mathrm{WD}$ vehicles, a ten degree of freedom nonlinear vehicle dynamics model is established and the torque distribution characteristic of an inter-wheel torque distribution device is analyzed. Based on the two degree of freedom linear vehicle model, the direct yaw moment controller with distribution of driving force is designed by means of sliding mode variable structure control. The active suspension system controller with a double-loop control structure is designed on the basis of its dynamics model. According to the analysis of the influence of the suspension on the vehicle steering performance, the integrated control of direct yaw moment control system and active suspension system is studied. The simulation of step steering condition and single lane change condition of vehicle is carried out using MATLAB/Simulink software. The simulation results show that the integrated control system can obviously improve vehicle riding comfort and handling stability much more than the individual control.

Key words: Four-wheel drive vehicle, direct yaw moment control system, active suspension system, integrated control, simulation.

Received April 08, 2016

Accepted June 08, 2017 